\title{
Earth's Shadowing Effects by Computer Algebra
}

\author{
S. D. Šegan
}

\begin{abstract}
The short-periodic perturbations of orbits of an artificial satellite due to the radiation pressure during one orbital period are influenced by Earth's shadow. For the semi-analytical theories it is necessary to calculate great number of coefficients. Using computer algebra we have computed them for some of applicable semi-analytical theories
\end{abstract}

Keywords: applied mathematics, informatics, mechanics

\section{Perturbing function and Earth's shadowing effects}

The perturbing acceleration due to the radiation pressure is given by equation

$$
\vec{f}=\vec{\kappa} P_{0} \frac{S}{m},
$$

where the vector $\vec{\kappa}$ directed from the Sun to the Earth, $S$ is satellite's cross-section, $m$ is mass of satellite and $P_{0}$ is numerical value of the solar pressure to the unit surface.

If the eccentric anomaly $E$ is taken as independent variable and if are used Lagrange perturbing equations for the variation of constants, after some algebraic transformations, the general formula for the arbitrary element $\rho$ can be obtained as

$$
\begin{gathered}
\frac{d \rho}{d E}=\frac{f_{p}^{\sigma}}{1-\cos E}\left[\left(1-e^{2}\right)^{s_{p}^{c}} \sum_{q=0}^{3} c_{p q}^{\sigma} \cos ^{q} E+\left(1-e^{2}\right)^{d_{p}^{c}}\right. \\
\left.\sum_{q=0}^{2} g_{p q}^{\sigma} \cos ^{q} E \sin E\right]
\end{gathered}
$$

where $f_{p}^{\sigma}$ is function of $f$ and some orbital elements, $c_{p q}^{\sigma}, g_{p q}^{\sigma}$ are functions of orbital elements and $s_{p}^{c}, d_{p}^{c}$ are some constants.

The powers of trigonometrical functions of $E$ can be transformed into functions of multiple of argument; method was used by [5] and [4] for computation of short-periodic perturbations due to atmospheric drag. Some parts of equation (2) are developed into infinite

Manuscript received November 22, 2020. ; accepted March 6, 2020.

S. D. Šegan is with the State University of Novi Pazar, Novi Pazar, Serbia 
power series which are convergent for $e<1$. In practical applications it is necessary to end the summation at the prior selected maximum of eccentricity $e_{\max }$ (see Appendix I).

This semi-analytical method has main advantage in possibility to produce perturbing coefficients and all expressions by computer algebra. The maximum power as function of $e_{\max }$ can be treated as an input parameter. In some simple cases, there is no necessity to use this rather complicated approach.

\section{Disturbing of radiation pressure perturbing function by Earth's shadow}

Until [1] has introduced a special "shadow function", which has zero value if the satellite is in shadow and equals one in the opposite case, the shadowing effect is taken into account numerically [2]. Later, [3] and [6] have introduced different form of shadow function with peculiar series expansion.

General lines of these shadow functions lies from the geometry given by Fig 1. Assume that Earth is spherical and shadow is cylindrical; on arbitrary satellite orbit, let angular distance of the satellite $\mathrm{S}$ from axis defined by direction across centers of Sun and Earth is $\lambda$ and angular semi-diameter of crossing point of momentary orbit with shadow is $\Phi$. The shadow function is

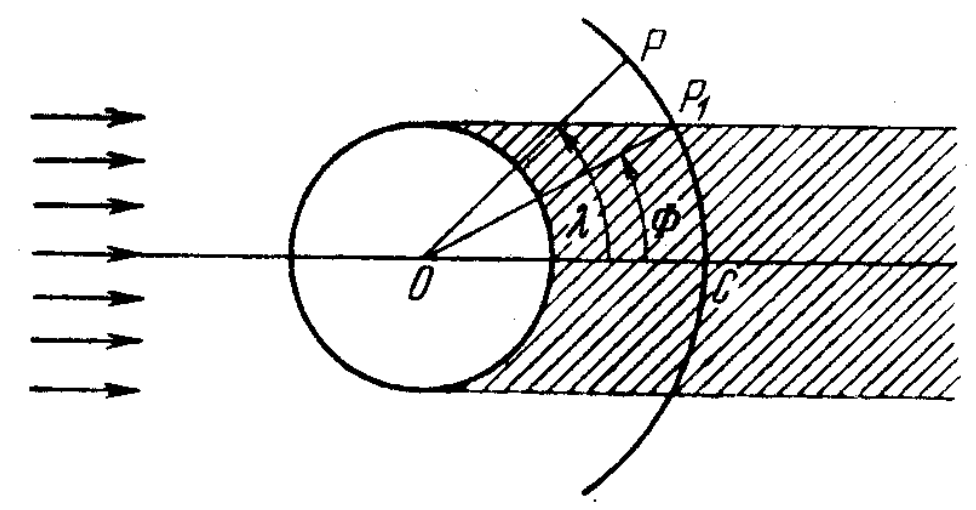

Fig. 1. The geometry of Earth's shadow effects

$$
\Psi= \begin{cases}0, & -\phi<\lambda<\Phi \\ 1, & -\pi<\lambda<-\Phi, \Phi<\lambda<\pi\end{cases}
$$

where $\sin \Phi=r\left(t_{0}\right) / R_{\oplus}$ and $r\left(t_{0}\right)$ is radius vector at moment of intersection with shadow boundary. By using this definition [1] is expanded $\Psi$ in Fourier's series:

$$
\Psi=\frac{a_{0}}{2}+\sum_{k=1}^{\infty} a_{k} \cos k \lambda
$$


where

$$
a_{k}=\frac{2}{\pi} \int_{0}^{\pi} \Psi \cos k \lambda d \lambda
$$

After dividing integration interval on $[0, \Phi]$ and $(\Phi, \pi]$ we have

$$
a_{0}=\frac{2}{\pi}(\pi-\Phi), \quad a_{k}=-\frac{2}{k \pi} \sin k \Phi .
$$

By introducing this in (5), we have

$$
\Psi=1-\frac{\Phi}{\pi}-\sum_{k=1}^{\infty} \frac{2}{k \pi} \sin k \Phi \cos k \lambda .
$$

The computation of the coefficients of this expansion is not so complicated, but after multiplying perturbation equation (2) for an arbitrary orbital element by (5) integration will be very complicated.

And, [3] was started from the fact that discontinuity in shadow function can be represented in dependance of main argument $x=\lambda-\Phi$ as

$$
\Psi=\frac{1}{2}\left(1+\frac{\sin x}{+\sqrt{1-\cos ^{2} x}}\right)
$$

which leads to

$$
\begin{gathered}
\Psi=\frac{1}{2}\left\{1+\sum_{j=0}^{\infty} \sum_{k=j}^{\infty} D_{j k} \sin ^{2 j+1}(\lambda-\Phi)\right\} \\
\Psi=\frac{1}{2}\left\{1+\sum_{j=0}^{\infty} \sum_{k=0}^{\infty} B_{j k} \sin ^{j} \Phi \cos ^{k} \lambda\right\}
\end{gathered}
$$

with

$$
D_{j k}=(-1)^{j} \frac{(2 k-1) ! !}{(2 k) ! !} C_{j k}
$$

where $C_{j k}$ are binomial coefficients (see Appendix II).

Comparison of [1] and [3] approximation of shadow function is given by Fig 2. for upper summation limit of 20.

So, [6] has expanded shadow function into series of Legendre polynomials (Appendix I)

$$
\Psi=\sum_{k=0}^{\infty} c_{k} P_{k}(\cos \lambda)
$$

By using $P_{k}(-1)=(-1)^{k}$ and known formula for calculation of the coefficients

$$
c_{k}=\frac{2 k+1}{2} \int_{0}^{\pi} \Psi P_{k}(\cos \lambda) \sin \lambda d \lambda
$$




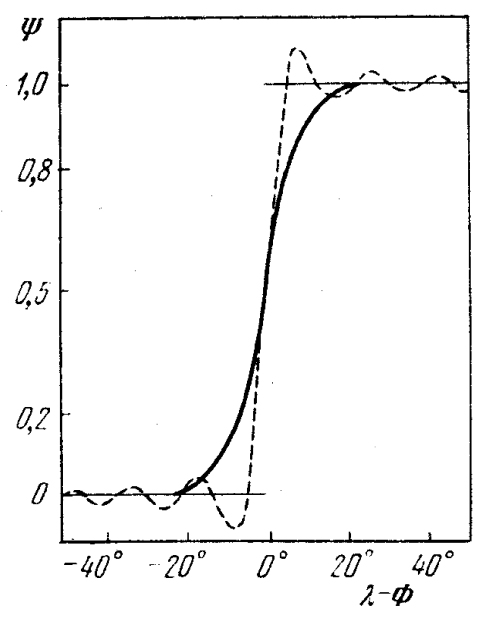

Fig. 2. figure caption 2

we have

$$
\begin{gathered}
\Psi=\frac{1}{2}(1+\cos \Phi)+\frac{1}{2} \sum_{k=1}^{\infty} P_{k}(\cos \lambda) P_{k+1}(\cos \Phi) \\
-\frac{1}{2} \sum_{k=1}^{\infty} P_{k}(\cos \lambda) P_{k-1}(\cos \Phi) .
\end{gathered}
$$

For upper limits of 10 and 20 [6] shadow function is shown on Fig 3.

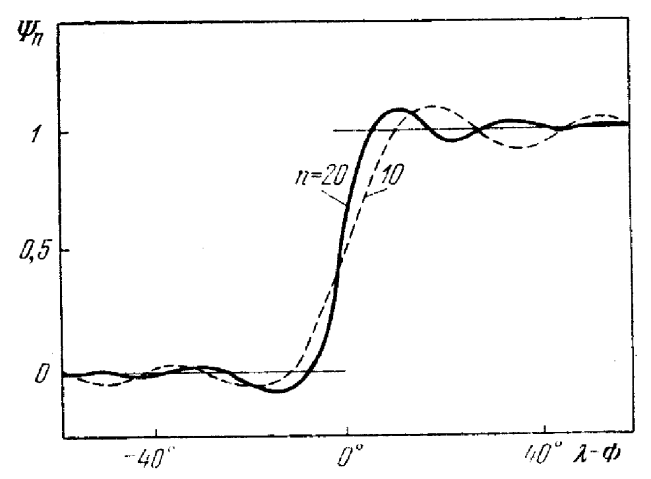

Fig. 3. Figure caption 3

Best geometric representation is given by [3] function. Calculation of necessary coefficients and integration of perturbation equations are simplest with [6] function. 


\section{Computer algebra procedures}

By integration of the Lagrange perturbation equations multiplied by some of shadow function we can develop "analytical" theory of satellite motion under influence of solar radiation pressure. But, first point is development above expressions and calculation of numerical coefficients. As $E$ is main argument, we need analytical expression for Kepler equation solution with arbitrary (choosen) number of terms.

In the case of [6] shadow function, convergence of series expansion is corrected by introducing exchange of indexes in first sum $k$ with $n-1$ and in second sum $k$ with $n-1$. After that, all $P_{n}(\cos \lambda)$ and $P_{n}(\cos \Phi)$ can be represented via orbital elements (see Appendix I).

In order to develop procedure to obtain Legendre polynomials and their application, we have used algebraic system REDUCE on IBM 390 computer. The procedure is described in the Appendix I.

For the [3] shadow function the quality of approximation depends on the number of terms used; development of expansion and calculation of coefficients are very difficult. By REDUCE system we obtain them after implementation some of procedures described as follows. Some suggestion regarding symmetry to main diagonal of matrix of coefficients was accepted from Z. Kalina (priv. comm.)

All developments are represented at appendices (I and II).

\section{Conclusions}

The given procedures for Legendre polynomials with arbitrary number of terms is basic for next step of the analytical integration of perturbation equation (2).

Similarly, the procedure in Appendix II is necessary for the definition of coefficients of the relation (8). As it be seen, the integration of the perturbing function is more complicated then with approximation by Legendre polynomials. Moreover, without computer algebra it is possible only under some additional assumptions regarding orbital characteristic.

Finally, our results suggest that it is advisable to develop new type, analytical more convenient and less complicated shadow function.

\section{Appendix I}

OUT IZLAZ;

COMMENT LEGENDRE POLYNOMIALS AND ASSOCI-

ATED FUNCTIONS;

COMMENT NUMBER (INDEX) BOUNDARY N;

$\mathrm{N}:=5 \$$

ARRAY PL $(\mathrm{N}), \mathrm{P}(\mathrm{N}, \mathrm{N})$;

FORALL FI LET $(1-\operatorname{SIN}(\mathrm{FI}) * * 2) * *(1 / 2)=\operatorname{COS}(\mathrm{FI}) ; 1) * * \mathrm{I}$;

$\mathrm{PL}(1):=1$

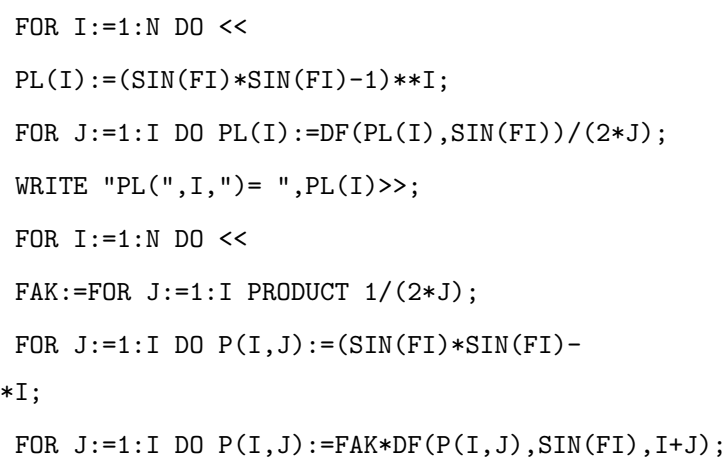


FOR J:=1: I DO FOR $\mathrm{K}:=1: \mathrm{J}$ DO $\mathrm{P}(\mathrm{I}, \mathrm{J}):=\mathrm{P}(\mathrm{I}, \mathrm{J}) *(1-$ $\operatorname{SIN}(\mathrm{FI}) * * 2) * *(1 / 2)$;

FOR J:=1:I DO WRITE "P(", I,",", J," )=

",P(I, J) >>;

SHUT IZLAZ;

BYE;

OUT IZLAZ;

OFF NAT; ON DIV;

WRITE "COMMENT N-BOUNDARY, ECC- ECCENTRIC-

ITY, AM- MEAN ANOMALY";

ALGEBRAIC PROCEDURE KEPLER (N,ECC,AM);

$\mathrm{EA}:=\mathrm{AM}+$

FOR $\mathrm{J}:=1: \mathrm{N}$ SUM

$\mathrm{ECC} * * \mathrm{~J} * \mathrm{DF}$ (SIN (AM) **J, AM, J-1) *

(FOR L:=1: J PRODUCT (1/L));

WRITE " EA(1)=",

$\operatorname{KEPLER}(1, \operatorname{ECC}, \mathrm{AM})$;

WRITE " EA(3)=",

$\operatorname{KEPLER}(3, \mathrm{ECC}, \mathrm{AM})$;

WRITE " $\operatorname{EA}(6)="$,

$\operatorname{KEPLER}(6, \mathrm{ECC}, \mathrm{AM})$;

WRITE " EA(8)=",

$\operatorname{KEPLER}(8, \mathrm{ECC}, \mathrm{AM})$;

SHUT IZLAZ;

END;

BYE;

\section{Appendix II}

OUT IZLAZ;

PROCEDURE MIMA(U,V); IF U $\mathrm{V}$ THEN U ELSE V; PROCEDURE BINKO $(\mathrm{N}, \mathrm{K})$;

BEGIN SCALAR BNK;

IF $N<K$ THEN WRITE

"+++ ERROR AT BINKO N=", N," $\mathrm{K}=", \mathrm{~K}$

ELSE GOTO OK; RETURN;

OK: IF $\mathrm{K}=1$ THEN BNK:=N ELSE $<<$

IF $(\mathrm{K}=\mathrm{N}$ OR $\mathrm{K}=0)$ THEN BNK: $=1$

ELSE BNK: $=\mathrm{FAC}(\mathrm{N}) /(\mathrm{FAC}(\mathrm{N}-\mathrm{K}) * \mathrm{FAC}(\mathrm{K}))>>$; RE-

TURN BNK; END;
PROCEDURE PBINKO $(\mathrm{X}, \mathrm{Y})$;

BEGIN SCALAR P; $P:=$

IF $Y<O$ THEN $O$ ELSE

IF DEN(Y) NEQ 1 THEN 0 ELSE

IF $\mathrm{Y}=0$ THEN 1 ELSE

(FOR I: $=0:(\mathrm{Y}-1)$ PRODUCT $(\mathrm{X}-\mathrm{I})) / \mathrm{FAC}(\mathrm{Y})$;

RETURN P; END;

PROCEDURE BJK (JCP, KCP, MJJ);

COMMENT $* * *$ DATUM $* * * \$$

BEGIN INTEGER Q,R,MJ, KO, J,L, M;

SCALAR SF;

$\mathrm{Q}:=\mathrm{JCP} ; \mathrm{R}:=\mathrm{KCP} ; \mathrm{MJ}:=\mathrm{MJJ}$;

$\operatorname{IF}(\operatorname{DEN}(Q / 2)=1$ AND DEN(R/2) NEQ 1) OR

$(\operatorname{DEN}(\mathrm{Q} / 2) \operatorname{NEQ} 1$ AND $\operatorname{DEN}(\mathrm{R} / 2)=1)$

THEN RETURN;

$\mathrm{KO}:=\mathrm{IF}$ DEN $(\mathrm{Q} / 2)=1$ THEN 0 ELSE 1 ;

$\mathrm{SF}:=0 ; \mathrm{J}:=0 ; \mathrm{L}:=\mathrm{J} ; \mathrm{M}:=\mathrm{KO}$;

SETF : $\mathrm{SF}:=\mathrm{SF} *(-1) * * \mathrm{~J} *(-1) * * \mathrm{~L} * \operatorname{PBINKO}(-1 / 2, \mathrm{~L}) * \mathrm{BINKO}(\mathrm{L}, \mathrm{J})$

$* \operatorname{BINKO}(2 * \mathrm{~J}+1, \mathrm{M}) * \operatorname{PBINKO}(\mathrm{J}-\mathrm{M} / 2+1 / 2,(\mathrm{Q}-\mathrm{M}) / 2)$

$* \operatorname{PBINKO}(\mathrm{J}-\mathrm{M} / 2+1 / 2,(\mathrm{R}-\mathrm{M}) / 2)$;

$M:=M+2 ; \quad \operatorname{IF} \quad M<=M I M A((2 * J+1), \operatorname{MIMA}(Q, R)) \quad$ THEN

GO TO SETF; M:=KO;

$\mathrm{L}:=\mathrm{L}+1 ;$ IF $\mathrm{L}<=\mathrm{MJ}$ THEN GO TO SETF;

$\mathrm{J}:=\mathrm{J}+1 ; \mathrm{L}:=\mathrm{J} ;$ IF $\mathrm{J}<=\mathrm{MJ}$ THEN GOTO SETF;

$\mathrm{SF}:=(-1) * *((\mathrm{Q}+\mathrm{R}) / 2) * \mathrm{SF}$;

RETURN SF;

END;

PROCEDURE SETCP (IQ, FQ, MXJ , FROM , CNT) ;

BEGIN ARRAY FAC (42); $\operatorname{FAC}(0):=1$;

FOR $I:=1:(2 * M X J+1)$ DO FAC $(I):=I * F A C(I-1) \$$

FOR $Q:=I Q: F Q$ DO

FOR $\mathrm{R}:=(2 * \mathrm{MXJ}+2+(\mathrm{FROM}-1) * 2-\mathrm{Q})$ STEP 2

UNTIL MIMA $(\mathrm{Q},(2 * \mathrm{MXJ}+2+(\mathrm{FROM}-1) * 2-\mathrm{Q}+(\mathrm{CNT}-$

1)*2)) DO

WRITE "CP (", Q, ", ", R, " )=", BJK ( $Q, R, M X J) \$$

END;

ARRAY CP $(18,18)$;

$\mathrm{CP}(0,0):=1 \$ \operatorname{CP}(6,6):=-53361 / 256 \$$

$\mathrm{CP}(7,5):=-22869 / 128 \$ \operatorname{CP}(7,7):=29403 / 32 \$$

$\mathrm{CP}(8,4):=-114345 / 1024 \$ \mathrm{CP}(8,6):=809415 / 1024 \$$

$\mathrm{CP}(8,3):=-2801525 / 16384 \$$

$\mathrm{CP}(9,3):=-12705 / 256 \$$ 
